Supporting Information:

\title{
Dynamic Transport Control of Colloidal Particles by Repeatable Active Switching of Solute Gradients
}

\author{
Dogyeong Ha, Sangjin Seo, Kyunghun Lee, and Taesung Kim** \\ Department of Mechanical Engineering, Ulsan National Institute of Science and \\ Technology (UNIST), 50 UNIST-gil, Ulsan 44919, Republic of Korea
}

\section{CORRESPONDENCE:}

Taesung Kim

Department of Mechanical Engineering

Ulsan National Institute of Science and Technology (UNIST)

50 UNIST-gil, Ulsan 44919, Republic of Korea

E-mail: tskim@unist.ac.kr

Tel: +82-52-217-2313

Fax: $+82-52-217-2449$ 


\section{Supplementary Figures}
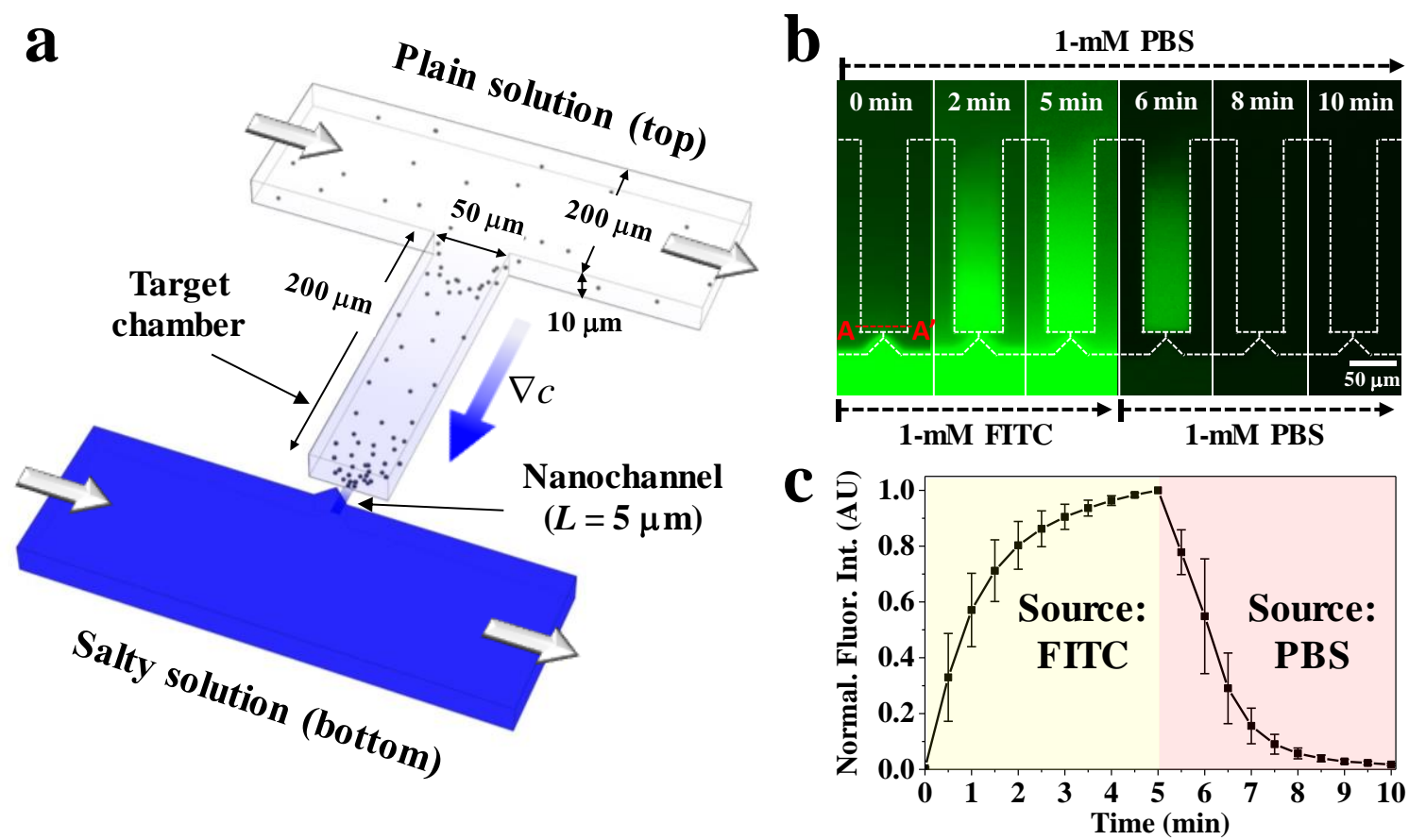

Figure S1. Characterization of the rate of diffusion through the nanochannel. (a) Schematic of the mixed-scale micro/nanofluidic diffusiophoresis (DP) platform (MNDP) for the generation of a solute concentration gradient. (b) Sequential fluorescence images show the formation of the fluorescein isothiocyanate (FITC) concentration gradient and its subsequent washing with phosphate-buffered saline (PBS) in the target chamber. (c) Graph shows the normalized fluorescence signal near the junction across the $\mathrm{A}-\mathrm{A}^{\prime}$ dashed line between the target chamber and nanochannel. The physicochemical environments completely switched from the FITC solution to a PBS solution by flushing the bottom channel by only using a pipette. 

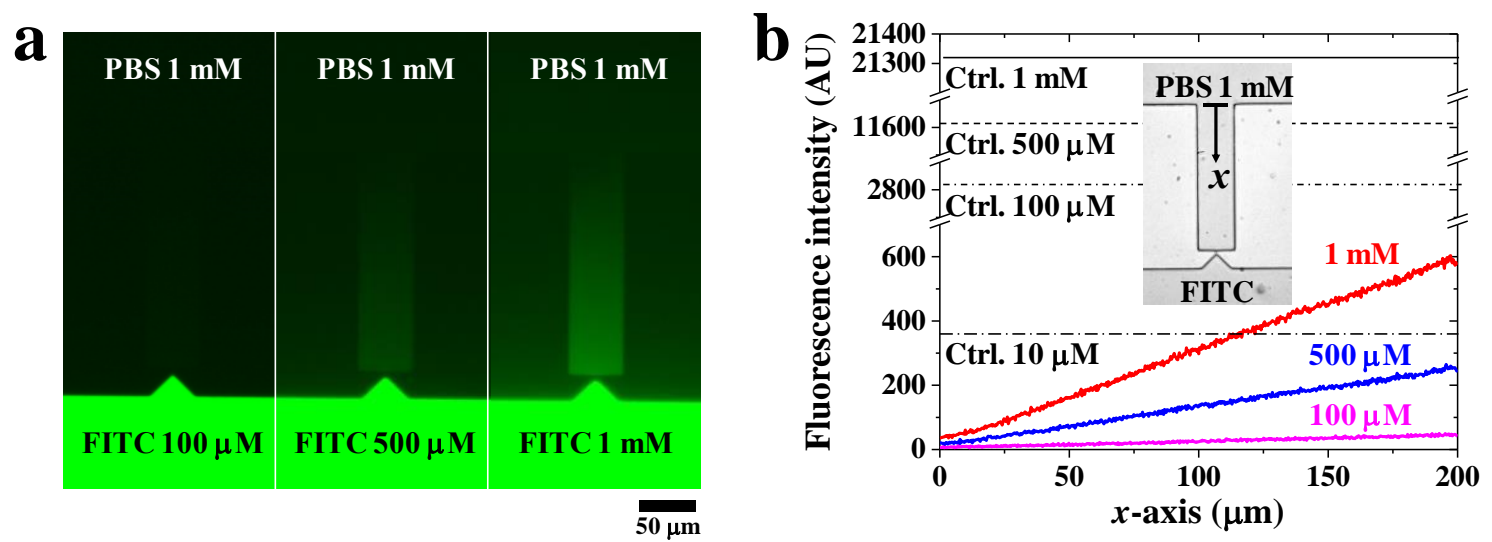

Figure S2. Characterization of the concentration gradient of target molecules in the MNDP. (a) Fluorescence images show the concentration gradient in the target chamber according to the FITC concentration, $c=100 \mu \mathrm{M}, 500 \mu \mathrm{M}$, and $1 \mathrm{mM}$ in the source channel, sequentially. (b) Quantification of the fluorescence signal in (a) and those of a reference value depending on the concentration in the source channel. FITC that passes through the nanochannel is largely diluted more than 10 times of the molecules in the source channel and is the reference fluorescence signal. 


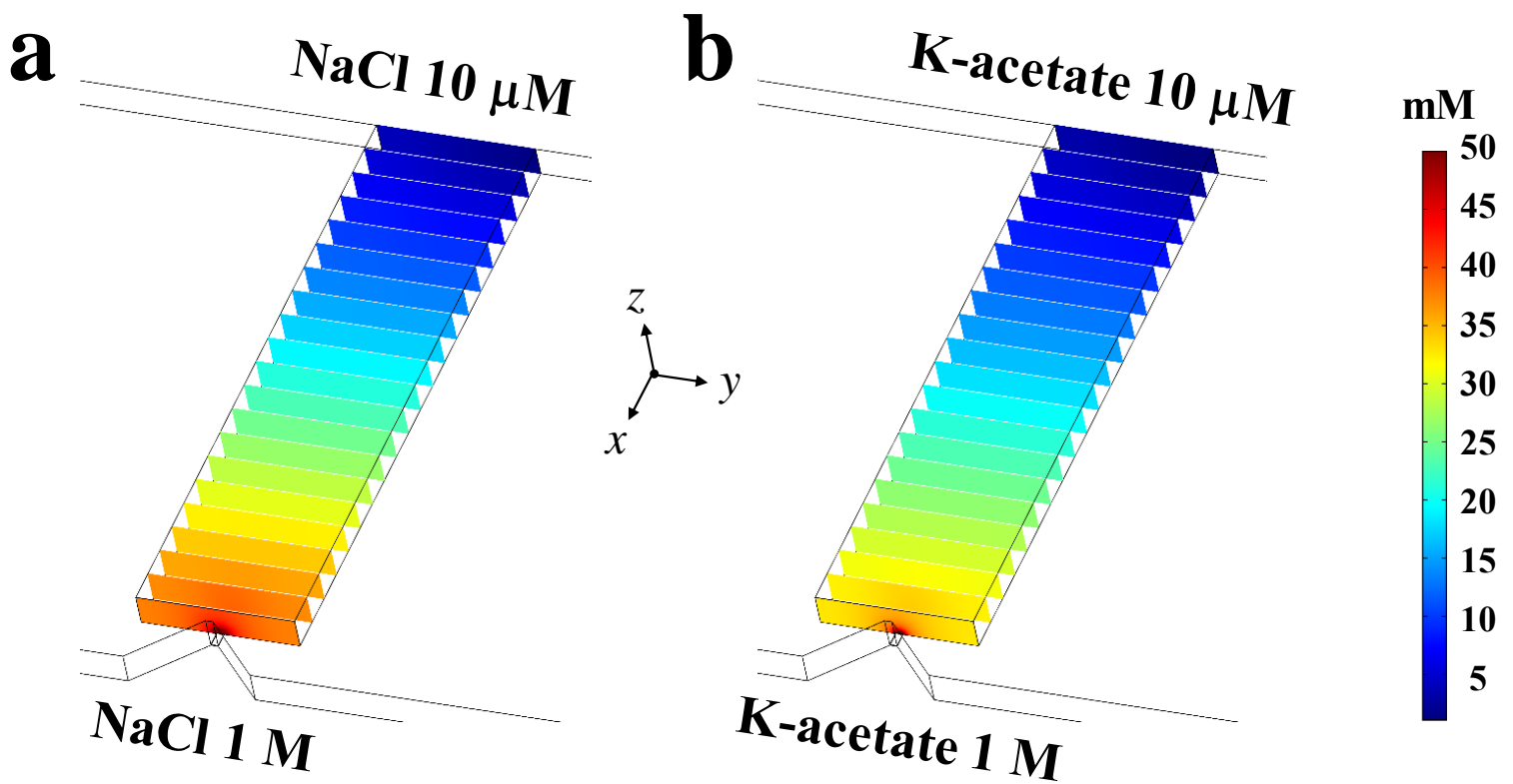

Figure S3. Numerical simulation results of the concentration gradient ( $y-z$ plane) at steadystate along the $x$-axis in the target chamber in the presence of (a) $\mathrm{NaCl}\left(c_{L}=10 \mu \mathrm{M}\right.$ and $c_{H}=1$ $\mathrm{M})$ and (b) K-acetate $\left(c_{L}=10 \mu \mathrm{M}\right.$ and $\left.c_{H}=1 \mathrm{M}\right)$, respectively. The fluid velocities in the source (bottom) and drain (top) channel are equally $200 \mu \mathrm{m} / \mathrm{s}$. 


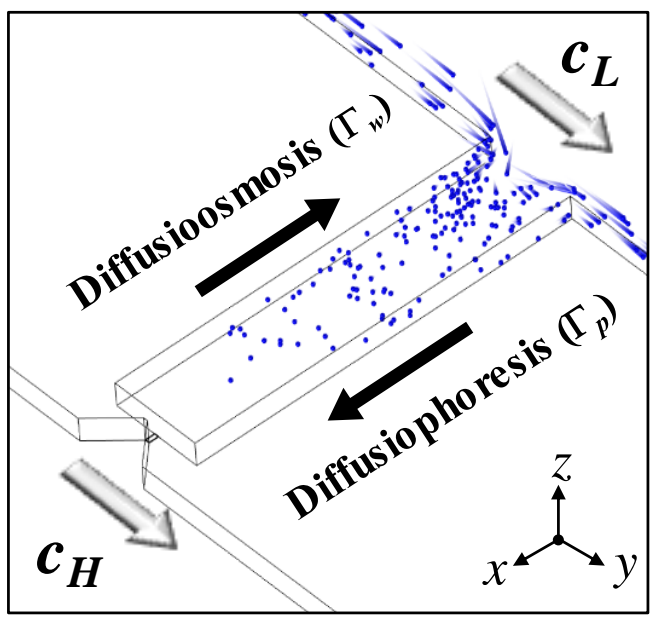

Figure S4. Perspective view of the numerical-simulation domain modeling the MNDP for tracing the particles $(0.05 \mathrm{w} / \mathrm{v} \%)$ in the presence of a solute concentration gradient of $\mathrm{NaCl}\left(c_{L}\right.$ $=10 \mu \mathrm{M}$ and $\left.c_{H}=1 \mathrm{M}\right)$. The dimensions of the domain are given in Figure S1. 


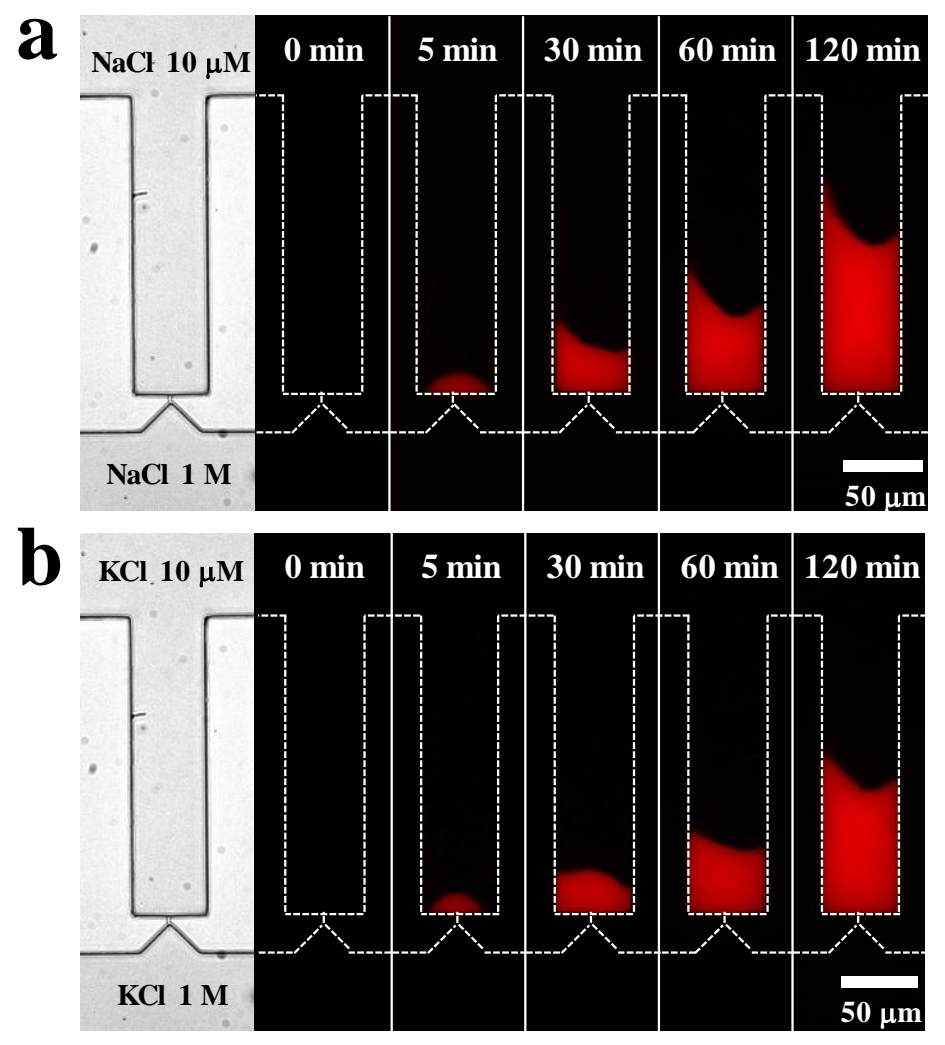

Figure S5. Sequential fluorescence images of the DP-based concentrations of colloidal particles within a period of $2 \mathrm{~h}$ in the presence of (a) $\mathrm{NaCl}$ concentration gradient $\left(c_{L}=10 \mu \mathrm{M}\right.$ and $\left.c_{H}=1 \mathrm{M}\right)$ and $(\mathbf{b}) \mathrm{KCl}$ concentration gradient $\left(c_{L}=10 \mu \mathrm{M}\right.$ and $\left.c_{H}=1 \mathrm{M}\right)$. 


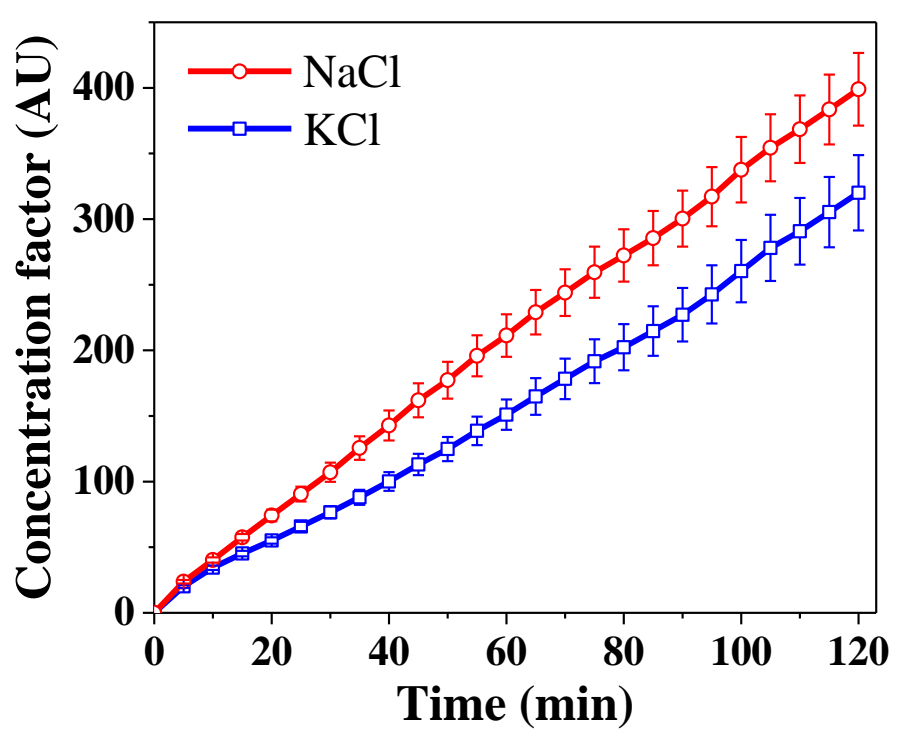

Figure S6. Concentration factors of the colloidal particles are quantified using the fluorescence intensities in the presence of the concentration gradients of $\mathrm{NaCl}$ and $\mathrm{KCl}\left(c_{L}=10 \mu \mathrm{M}\right.$ and $c_{H}$ $=1 \mathrm{M})$. As the diffusivity difference of $\mathrm{NaCl}\left(\beta_{\mathrm{NaCl}}=-0.207\right)$ is greater than that of $\mathrm{KCl}\left(\beta_{K C l}\right.$ $=-0.019)$, the concentration gradient of $\mathrm{NaCl}$ produces a relatively strong electric field compared to that of $\mathrm{KCl}$. 

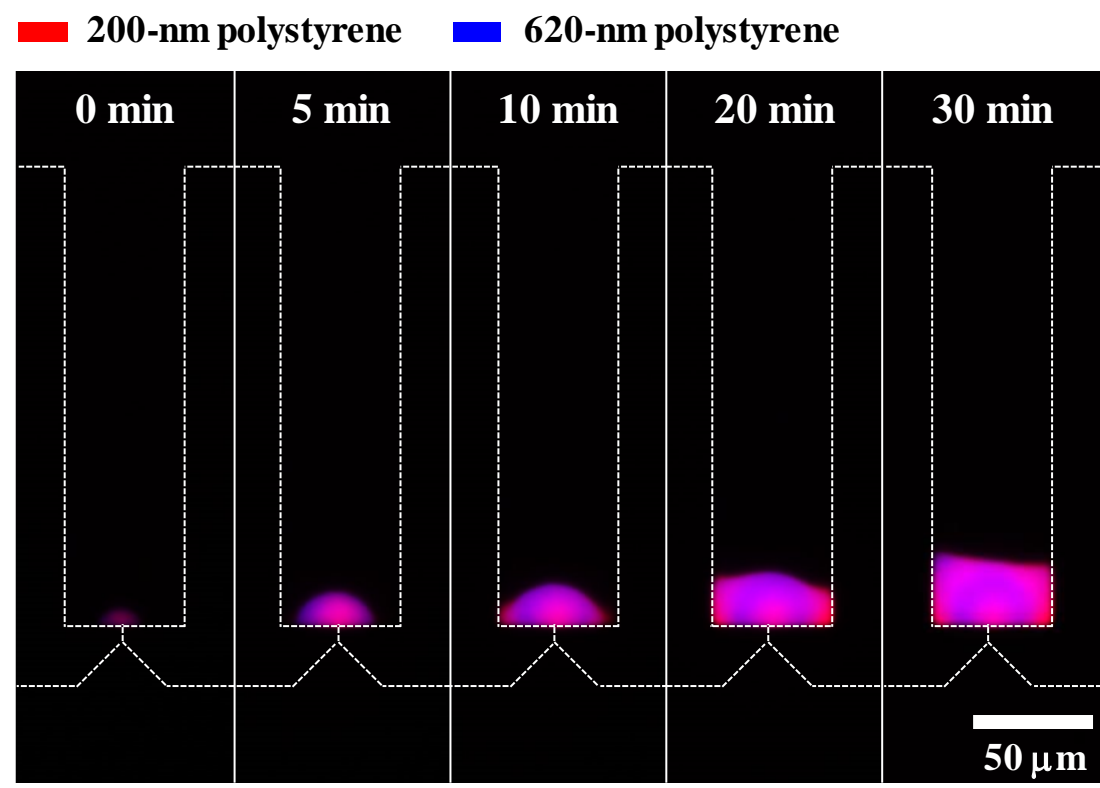

Figure S7. Sequential fluorescence images of the DP-based concentration of a nanoparticle mixture in the presence of the $\mathrm{NaCl}$ concentration gradient $\left(c_{L}=10 \mu \mathrm{M}\right.$ and $\left.c_{H}=1 \mathrm{M}\right)$ for 30 $\min$. 


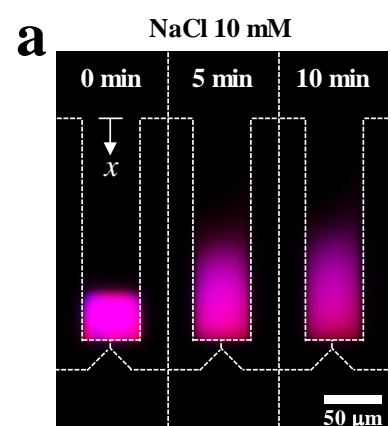

$\mathrm{NaCl} 10 \mathrm{mM}$

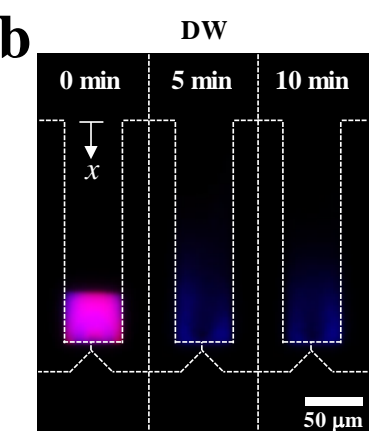

K-acetate 1 M

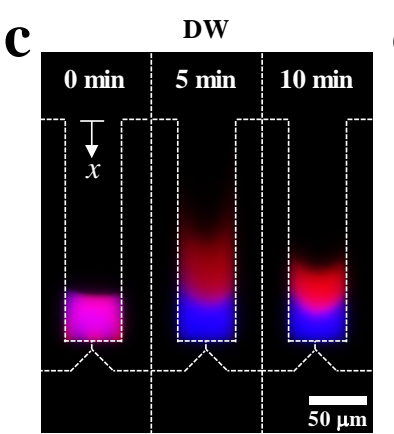

Na-acetate 1 M

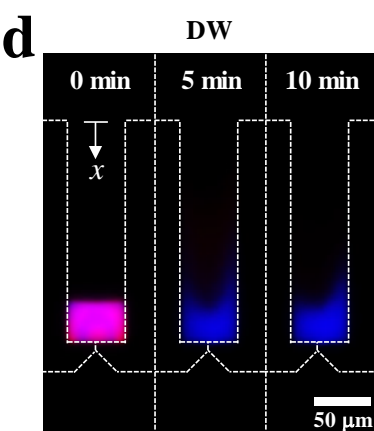

Na-acetate $800 \mathrm{mM}$ K-acetate $200 \mathrm{mM}$
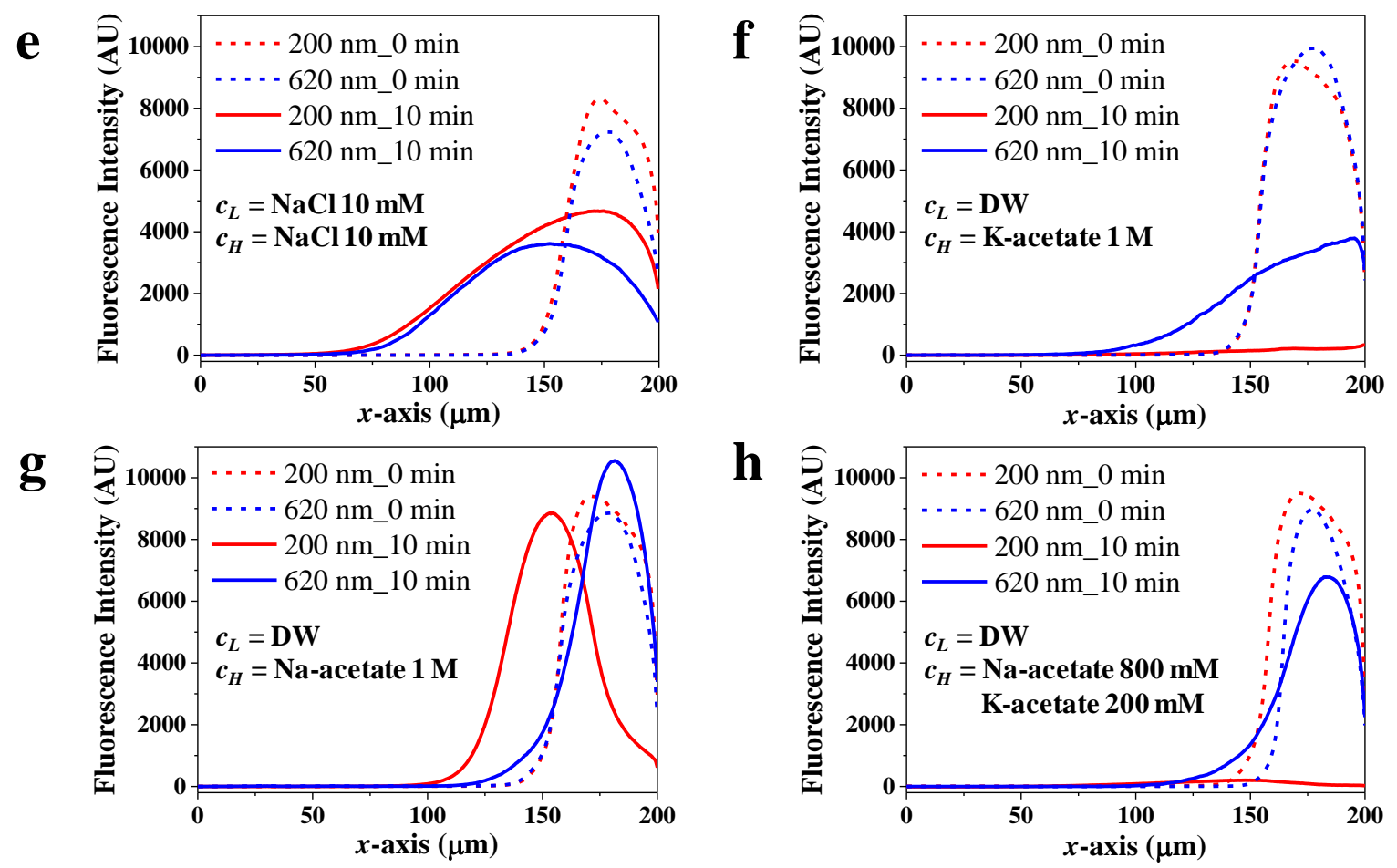

Figure S8. Motions of a submicron particle mixture consisting of red-fluorescent 200-nm and blue-fluorescent $620-\mathrm{nm}$ polystyrene particles. (a) The particles in the target chamber scatter over time by Brownian motion in the absence of the solute gradients (e.g., $c_{L}=c_{H}=10 \mathrm{mM}$ of $\mathrm{NaCl}$ ). The particles are separated and/or fractionated in the presence of the concentration gradient of (b) K-acetate ( $c_{L}=0 \mathrm{M}$ and $c_{H}=1 \mathrm{M}$ ), (c) Na-acetate $\left(c_{L}=0 \mathrm{M}\right.$ and $\left.c_{H}=1 \mathrm{M}\right)$, and (d) a mixture of Na-acetate $\left(c_{L}=0 \mathrm{M}\right.$ and $\left.c_{H}=800 \mathrm{mM}\right)$ and $\mathrm{K}$-acetate $\left(c_{L}=0 \mathrm{M}\right.$ and $c_{H}=200$ $\mathrm{mM}$ ). (e-h) Quantitative distribution of the particles at $t=0 \mathrm{~min}$ (dotted line) and $t=10 \mathrm{~min}$ (solid line) for the qualitative results of $(\mathrm{a}-\mathrm{d})$, respectively. 


\section{Supplementary Table}

Table S1. Zeta potentials of colloidal particles adjusted by Henry's function for various solution conditions measured through electrophoretic light scattering.

\begin{tabular}{lcccc}
\hline Particle & $E L S, \zeta_{p}(\mathrm{mV})$ & $f^{1}$ & $\zeta_{p}(\mathrm{mV})$ & Solution Condition $^{2}$ \\
\hline \multirow{2}{1\mu\mathrm{m},}{} & $-50.5 \pm 8.6$ & 1 & $-50.5 \pm 8.6$ & $\mathrm{NaCl}$ \\
Carboxylated & $-51.2 \pm 10.0$ & 1 & $-51.2 \pm 10.0$ & $\mathrm{KCl}$ \\
Polystyrene & $-53.5 \pm 8.4$ & 1 & $-53.5 \pm 8.4$ & K-acetate \\
& $-17.3 \pm 5.5$ & 1 & $-17.3 \pm 5.5$ & K-acetate \\
& & & & $0.02 \%$ Pluronic F-127 \\
\hline \multirow{2}{*}{$200 \mathrm{~nm}}$, & $-60.2 \pm 7.8$ & 0.828 & $-72.7 \pm 6.3$ & NaCl \\
Polystyrene & $-58.8 \pm 7.9$ & 0.836 & $-70.3 \pm 6.6$ & K-acetate \\
& $-58.4 \pm 7.2$ & 0.837 & $-69.8 \pm 6.0$ & Na-acetate \\
\hline \multirow{2}{*}{$620 \mathrm{~nm}}$, & $-68.6 \pm 5.9$ & 0.926 & $-74.1 \pm 5.4$ & NaCl \\
Polystyrene & $-67.8 \pm 5.2$ & 0.928 & $-73.1 \pm 4.9$ & K-acetate \\
& $-67.0 \pm 5.4$ & 0.929 & $-72.2 \pm 5.0$ & Na-acetate \\
\hline
\end{tabular}

${ }^{1}$ Henry's function is negligible $(f=1)$ for $1-\mu$ m particles.

${ }^{2}$ The concentration and $\mathrm{pH}$ of the solution are $10 \mathrm{mM}$ and 7.0, respectively. 
Table S2. Potential barrier energy for particle interactions

\begin{tabular}{|c|c|c|c|c|}
\hline Concentration & $200-200(\mathrm{~nm})$ & $200-620(\mathrm{~nm})$ & $620-620(\mathrm{~nm})$ & $1-1(\mu \mathrm{m})$ \\
\hline $1 \mathrm{mM}$ & 468 & 723 & 1447 & 1127 \\
\hline $10 \mathrm{mM}$ & 420 & 662 & 1300 & 958 \\
\hline $100 \mathrm{mM}$ & 337 & 556 & 1042 & 674 \\
\hline
\end{tabular}

Unit: $k_{B} T\left(\approx 0.411 \times 10^{-20} \mathrm{~J}\right)$. In addition, the zeta potentials are $\zeta_{1}=\zeta_{2}=-70 \mathrm{mV}$ and $\zeta_{1}{ }^{*}=\zeta_{2}{ }^{*}$

$=-50 \mathrm{mV}$ for polystyrene particles (i.e., 200 and $620 \mathrm{~nm}$ ) and carboxylated particles (i.e., 1 $\mu \mathrm{m})$, respectively. 


\section{Supplementary Methods}

\section{Characterization of the MNDP}

Supplementary Figure S1 shows the characterization of the diffusion rate in the target channel via a gradient of FITC. We introduced 1-mM FITC molecules dissolved in a 1-mM PBS solution to the bottom source channel and a pure 1-mM PBS solution containing no FITC to the top main channel. After measuring the FI along the $\mathrm{A}-\mathrm{A}^{\prime}$ line for $5 \mathrm{~min}$, we changed the FITC solution in the bottom source channel to pure PBS to confirm the time required to change the physicochemical environment in the target channel by measuring the FI at the same position.

\section{Reusability test of the MNDP}

Supplementary Movie S6 shows the reusability test of the MNDP. We utilized the same conditions for the preconcentration of the colloidal particles by using the concentration gradient of $\mathrm{NaCl}$ for $1 \mathrm{~h}$. After preconcentration, we changed the physicochemical condition to the same condition used for the full extraction of the preconcentrated colloidal particles by using the concentration gradient of K-acetate containing Pluronic F-127 for 15 min. The aforementioned steps for concentration and extraction were performed in a single cycle, which was repeated three times consecutively. We used bright-field observations for continuous recording of the experiment, including the buffer-change step (black image) in each cycle. In this setup, the extraction process in each cycle enables the evacuation of the target chamber, leaving only a few colloidal particles nonspecifically bound to the PDMS surface. 


\section{Supplementary Theory and Modeling}

\section{Dynamics of the fluid advection in the presence of solute gradient}

Fluid advection $\boldsymbol{v}_{f}$ was produced by Poiseuille and diffusioosmotic flows induced by the solute concentration gradients in the target chamber. ${ }^{1,2}$ To solve the Poiseuille flow, we introduced the Navier-Stokes and continuity equations describing the motions of incompressible flow as follows:

$$
\begin{gathered}
\rho \frac{\partial \boldsymbol{u}}{\partial t}+\rho(\boldsymbol{u} \cdot \nabla) \boldsymbol{u}=-\nabla p+\eta \nabla^{2} \boldsymbol{u}, \\
\nabla \cdot \boldsymbol{u}=0,
\end{gathered}
$$

where $\rho$ is the density and $\eta$ is the dynamic viscosity of the solution.

The diffusioosmotic flow is derived from the wall slip velocity, which is induced by the solute concentration gradient at the charged plane of the wall. The wall slip velocity is defined as $\boldsymbol{v}_{f, w}=-\Gamma_{w} \nabla \ln c$, where $\Gamma_{w}$ is the diffusioosmotic mobility and $c$ is the solute concentration. As the electrical double layer (EDL) of the wall is very thin (i.e., EDL thickness $\kappa^{-1}=\sim 3.07$ $\mathrm{nm})$ in a $10 \mathrm{mM}$ monovalent solution, $\Gamma_{w}$ takes the following form ${ }^{1}$ :

$$
\Gamma_{w}=\frac{\varepsilon}{\eta}\left(\frac{k_{B} T}{z e}\right)^{2}\left[\beta\left(\frac{z e \zeta_{w}}{k_{B} T}\right)+4 \ln \left(\cosh \frac{z e \zeta_{w}}{4 k_{b} T}\right)\right],
$$

where $\varepsilon$ is the permittivity of the medium, $\zeta_{w}$ is the zeta potential of the walls, $k_{B}$ is the Boltzmann constant, $T$ is the solution temperature, $z$ is the solute valence, and $e$ is the elementary electric charge. Nondimensionalized factor $\beta$ is defined as the diffusivity difference between cations and anions (defined in the main manuscript). The first and second terms of equation (3) represent electroosmosis (EP) and chemiosmosis (CP), respectively. 
Recall that wall slip velocity is in proportion to the concentration gradient of solutes. Therefore, the Nernst-Planck equation, expressed as follows, is used for solving the distribution of solutes:

$$
\boldsymbol{j}_{i}=-\left[D_{i} \nabla c_{i}-\boldsymbol{u} c_{i}+\frac{D_{i} z_{i} e}{k_{B} T} c_{i} \nabla \phi\right],
$$

where $\boldsymbol{j}_{i}, D_{i}, z_{i}$, and $c_{i}$ are the flux, diffusivity, valence, and concentration of each species, respectively, and $\phi$ is the electric potential. As the total current should be zero, assuming local charge neutrality, equation (4) can be rewritten as the advection-diffusion equation for $z=z+=$ $-z$ - electrolytes with ambipolar diffusivities, ${ }^{1,3}$ which are respectively defined as follows:

$$
\begin{gathered}
\boldsymbol{j}_{+}=\boldsymbol{j}_{-}=-\left[D_{s} \nabla c-\boldsymbol{u} c\right] \\
D_{s}=\frac{2 D_{+} D_{-}}{D_{+}+D_{-}} .
\end{gathered}
$$

As the governing equations are coupled, a numerical multiphysics simulation was performed to calculate the particle motion in the presence of a solute gradient.

\section{Zeta-potential measurement for size-dependent particles}

The electrophoretic mobility of particles was measured using electrophoretic light scattering (Malvern Panalytical Zetasizer Nano ZS). We obtained the zeta potential of particles from the measured electrophoretic mobility considering Henry's function dealing with the retardation effect, when the particle radius $a$ is comparable to EDL thickness $\kappa^{-1}(\kappa a \gg 10)$, as described in a previous study. ${ }^{3}$

$$
\Gamma_{E P}=f \frac{\varepsilon \zeta}{\eta}\left(\frac{2}{3}<f<1\right)
$$


where $f=f(\kappa a)$ is Henry's function, $f=1$, for $\kappa a \rightarrow \infty$ and $f=2 / 3$ for $\kappa a \rightarrow 0$.

For $\kappa a \gg 10$ and symmetric electrolytes with valence of $z$, analytical approximation for Henry's function is given as ${ }^{3}$

$$
\begin{aligned}
& f(\kappa a)=1-\left(\frac{2 k_{B} T}{z e\left|\zeta_{p}\right|}\right)\left(\frac{F H}{1+F}\right)+\left(\frac{k_{b} T}{z e\left|\zeta_{p}\right|}\right)\left(\frac{1}{\kappa a}\right)\left[-12 K\left(t+\frac{t^{3}}{9}\right)\right. \\
& +\left(\frac{10 F}{1+F}\right)\left(t+\frac{7 t^{3}}{20}+\frac{t^{3}}{9}\right)-4 G\left(1+\frac{3}{D_{-}^{*}}\right)\left(1-\exp \left(-\frac{z e\left|\zeta_{p}\right|}{2 k_{B} T}\right)\right) \\
& +\frac{8 F H}{(1+F)^{2}}+\left(\frac{6 z e\left|\zeta_{p}\right|}{k_{B} T}\right)\left(\frac{1}{1+F}\right)\left(\frac{G}{D_{-}^{*}}+\frac{H}{D_{+}^{*}}\right) \\
& \left.-\left(\frac{24 F}{1+F}\right)\left(\frac{G^{2}}{D_{-}^{*}}+\frac{H^{2}}{(1+F) D_{+}^{*}}\right)\right]
\end{aligned}
$$

Here, $D_{ \pm}^{*}=\frac{3 \eta z^{3} e^{2}}{2 \varepsilon k_{B}^{2} T^{2}} D_{ \pm}$is the dimensionless diffusivity for the anions $\left(D_{-}^{*}\right)$ and cations $\left(D_{+}^{*}\right)$, and $t, F, G, H$, and $K$ are

$$
\begin{gathered}
t=\tanh \left(\frac{z e\left|\zeta_{p}\right|}{4 k_{B} T}\right), F=\frac{2}{\kappa a}\left(1+\frac{3}{D_{ \pm}^{*}}\right)\left(\exp \left(\frac{z e\left|\zeta_{p}\right|}{2 k_{B} T}\right)-1\right) \\
G=\ln \left(\frac{1+\exp \left(-\frac{z e\left|\zeta_{p}\right|}{2 k_{B} T}\right)}{2}\right), H=\ln \left(\frac{1+\exp \left(\frac{z e\left|\zeta_{p}\right|}{2 k_{B} T}\right)}{2}\right) \\
K=1-\frac{25}{3(10+\kappa a)} \exp \left[-\frac{z e\left|\zeta_{p}\right|}{6 k_{B} T}\left(\frac{\kappa a}{6+\kappa a}\right)\right]
\end{gathered}
$$

Zeta potential of colloidal particles measured through electrophoretic light scattering and calculated using Henry's function are shown in Table S1. 


\section{Derjaguin-Landau-Verwey-Overbeek (DLVO) theory for stability analysis}

The DLVO theory explains the stability of colloidal particles in case of aggregation due to the combined effect of van der Waals' and electrostatic interactions. ${ }^{4}$ As the DP-induced concentration of the colloidal particles narrows the distances between particles, an irreversible particle aggregation could occur depending on the value of energy barrier. To elucidate the stability issue in a quantitative manner, readers can refer to literature. ${ }^{4}$ For two unequal spheres, van der Waals' interaction energy $\left(V_{A}\right)$ between unequal particles in water was considered:

$$
V_{A}=-\frac{A_{12}}{12}\left[\frac{y}{x^{2}+x y+x}+\frac{y}{x^{2}+x y+x+y}+2 \ln \left(\frac{x^{2}+x y+x}{x^{2}+x y+x+y}\right)\right],
$$

where $A_{12}$ is the Hamaker constant, and $x=h / d_{1}$ and $y=d_{2} / d_{1}$ are defined when $h$ is the distance between two particles, and $d_{1}$ and $d_{2}$ are the diameters of two particles. The Hamaker constant of polystyrene spheres in water is suggested as $A_{12}=0.95 \times 10^{-20} \mathrm{~J}$. For a very short distance $(x \ll 1)$, equation $(9)$ is reduced to

$$
V_{A}=-\frac{A_{12}}{12 h} \frac{d_{1} d_{2}}{d_{1}+d_{2}}
$$

For unequal two spheres, the EDL interaction energy $\left(V_{R}\right)$ was introduced through Derjaguin's method.

$$
V_{R}=2 \pi \varepsilon \zeta_{1} \zeta_{2} \frac{d_{1} d_{2}}{d_{1}+d_{2}} \exp (-\kappa h)
$$

where $\varepsilon$ is the permittivity of the medium, $\zeta_{1}$ and $\zeta_{2}$ are the zeta potential of two particles, and $\kappa^{-1}$ is the EDL thickness. Therefore, the total interaction energy is given as follows: 


$$
V_{T}=V_{A}+V_{R}
$$

Here, potential barrier energy $\left(V_{\mathrm{T}, \max }\right)$ can be a reference of the particle stability. We assumed $\zeta_{1}=\zeta_{2}=-70 \mathrm{mV}$ for polystyrene particles (i.e., for 200 and $620 \mathrm{~nm}$ ) and $\zeta_{1}{ }^{*}=\zeta_{2}{ }^{*}=-50 \mathrm{mV}$ for carboxylated particles (i.e., $1 \mu \mathrm{m}$ ), respectively. We measured the zeta potential of the particles as shown in Table $\mathrm{S} 1$. The concentrations of $c=1,10$, and $100 \mathrm{mM}$ represent the range of concentrations in the microchamber and were estimated using the simulation result in Figure S3. The calculated potential barrier energy for all four cases is shown in Table S2. For all concentrations and particle sizes, the potential barrier energy has an even larger value than the general requirement (i.e., $25 k_{B} \mathrm{~T}$ ) for the stable state. ${ }^{4}$ Therefore, the colloidal particles used in this study may not go through irreversible aggregation.

\section{Numerical method}

COMSOL Multiphysics (ver 5.3), the commercial finite-element-modeling software, was used to solve the governing equations. Correspondingly, a creeping-flow module, transport-ofdiluted-species module, and particle-tracing module were used. The creeping-flow module solves the Navier-Stokes equation, neglecting the inertial term (Stokes flow) for the conservation of momentum and continuity for mass conservation in fluid flow. The transportof-diluted-species module solves the advection-diffusion equation for solute concentration. The solute concentration gradient induces diffusioosmotic flow along the walls of the target chamber and nanochannel. Hence, the fluid flow and solute flux were coupled. The particletracing module visualizes the trace of individual particles with velocity $\boldsymbol{v}_{p}$, which is the sum of flow velocity $\boldsymbol{v}_{f}$ and diffusiophoretic velocity $\boldsymbol{v}_{D P}$. 
The numerical model of the MNDP comprises the top main channel, bottom source channel, target chamber, and nanochannel, as shown in Figure S2. The top main channel and bottom source channel are geometrically equal, and their dimensions in the numerical domain are $400 \mu \mathrm{m}$ (length) $\times 200 \mu \mathrm{m}$ (width) $\times 10 \mu \mathrm{m}$ (height). The target chamber is $200-\mu \mathrm{m}$ long, $50-\mu \mathrm{m}$ wide, and $10-\mu \mathrm{m}$ high, whereas the nanochannel positioned at the surface of the substrate is $5-\mu \mathrm{m}$ long, $1.5-\mu \mathrm{m}$ wide, and $200-\mathrm{nm}$ high. For these dimensions, the diffusion toward the direction opposing flow in the top main channel is negligible. That is, $P e$ is estimated to be 24.8 and 28.6 for $\mathrm{NaCl}$ and $\mathrm{K}$-acetate, respectively, when the length scale is $200 \mu \mathrm{m}$, average velocity is approximately $200 \mu \mathrm{m} / \mathrm{s}$, and ambipolar diffusivity of each electrolyte is assumed. Then, the top main channel is designed to introduce low concentrations of $\mathrm{NaCl}\left(c_{L}=10 \mu \mathrm{M}\right)$ and $\mathrm{K}$-acetate containing particles for the concentration and extraction processes, respectively. The bottom source channel is designed to introduce high concentrations of $\mathrm{NaCl}\left(c_{H}=1 \mathrm{M}\right)$ and $\mathrm{K}$-acetate for the concentration and extraction, respectively. Only the top main channel contains particles.

We performed numerical simulations for the particle concentration and extraction to validate our experiments and further investigate the wall effect. First, the concentration of particles in the presence of the $\mathrm{NaCl}$ gradient is shown in Supplementary Movie S3. The concentration gradient of $\mathrm{NaCl}$ is initially produced by introducing a $10-\mu \mathrm{M} \mathrm{NaCl}$ solution to the top main channel $\left(c_{L}\right)$ and a 1-M NaCl solution to the bottom source channel $\left(c_{H}\right)$. Subsequently, the solution in the top main channel is changed to the solution of colloidal particles with $10-\mu \mathrm{M} \mathrm{NaCl}$. We imposed the solution velocities for both channels at the inlets as $200 \mu \mathrm{m} / \mathrm{s}$, as experimentally measured according to the particle velocity in the top main 
channel. We also imposed wall-slip boundary conditions with respect to the diffusioosmotic flow $\left(\Gamma_{w}=780 \mu \mathrm{m}^{2} / \mathrm{s}\right)$ provided by the known zeta potential of the PDMS wall treated with oxygen plasma $\left(\zeta_{w}=-72 \mathrm{mV}\right)$ in the $5-\mathrm{mM} \mathrm{NaCl}$ solution. ${ }^{3}$ The diffusiophoretic velocity of the particle is given by the diffusiophoretic mobility $\left(\Gamma_{p}=450 \mu \mathrm{m}^{2} / \mathrm{s}\right)$ with respect to the measured zeta potential of the particle $\left(\zeta_{p}=-50.5 \mathrm{mV}\right.$ for $1-\mu \mathrm{m}$ carboxylated polystyrene, as shown in Table S1). Second, the preconcentrated particles were extracted in the presence of the steady-state concentration gradients of pure K-acetate and K-acetate containing Pluronic F127, as shown in Supplementary Movies S4 and S5, respectively. The simulation results of the extraction of the preconcentrated particles in the steady state show good agreement with the experimental results. We ignored the effect of an interdiffusion phenomenon between $\mathrm{NaCl}$ and K-acetate, which can be significant for the real situations, in particular, for the transient state (i.e., during solution change).

In addition, we assumed that the presence of the surfactant affects only the zeta potentials of the wall and particles, and thus the initial boundary conditions were set to be equal for both cases. First, colloidal particles initially accumulated in the target chamber in the presence of the concentration gradient of K-acetate $\left(c_{H}=1 \mathrm{M}\right.$ and $\left.c_{L}=10 \mu \mathrm{M}\right)$. We imposed the diffusiophoretic mobility for pure $\mathrm{K}$-acetate $\left(\Gamma_{p}=-40 \mu \mathrm{m}^{2} / \mathrm{s}\right)$ and $\mathrm{K}$-acetate containing Pluronic F-127 $\left(\Gamma_{p}=-70 \mu \mathrm{m}^{2} / \mathrm{s}\right)$ with the measured zeta potential of the particle $\left(\zeta_{p}=-53.5\right.$ $\mathrm{mV}$ for pure $\mathrm{K}$-acetate and $\zeta_{p}=-17.3 \mathrm{mV}$ for $\mathrm{K}$-acetate containing Pluronic $\left.\mathrm{F}-127\right)$, as shown in Table S1. For the wall-slip boundary condition, instead of using the zeta potential of PDMS walls as used in literature, we used a properly estimated zeta potential value because measured wall zeta potentials show large discrepancies among different studies. ${ }^{3}$ Although the 
diffusioosmotic mobility is not a critical factor for particle concentration, a small variation in the diffusioosmotic mobility can significantly affect the extraction of the preconcentrated particles. Thus, we imposed the zeta potential of the PDMS wall for both cases, yielding relatively strong diffusioosmotic mobility for pure K-acetate (i.e., $\Gamma_{w}=-50 \mu \mathrm{m}^{2} / \mathrm{s}$ for $\zeta_{w}=-50$ $\mathrm{mV}$ ) and relatively weak diffusioosmotic mobility for K-acetate containing Pluronic F-127 (i.e., $\Gamma_{w}=-48 \mu \mathrm{m}^{2} / \mathrm{s}$ for $\zeta_{w}=-10 \mathrm{mV}$ ). 


\section{Supplementary Movies}

Movie S1: Transport of colloidal particles (1- $\mu \mathrm{m}$ carboxylated polystyrene) with different flow rates between the top main channel $\left(Q_{m}\right)$ and bottom source channel $\left(Q_{s}\right)$ in the absence of a solute concentration gradient. (a) $Q_{m}=50 \mathrm{~nL} / \mathrm{min}, Q_{s}=100 \mathrm{~nL} / \mathrm{min}$; (b) $Q_{m}=100 \mathrm{~nL} / \mathrm{min}, Q_{s}=$ $100 \mathrm{~nL} / \mathrm{min}$; and (c) $Q_{m}=500 \mathrm{~nL} / \mathrm{min}, Q_{s}=100 \mathrm{~nL} / \mathrm{min}$. Play speed is $2 \times$.

Movie S2: Transport of colloidal particles (1- $\mu \mathrm{m}$ carboxylated polystyrene) with the same flow rates between the top main channel and bottom source channel $\left(Q_{m}=Q_{s}=100 \mathrm{~nL} / \mathrm{min}\right)$ in the presence of the $\mathrm{NaCl}$ concentration gradient $\left(c_{L}=10 \mu \mathrm{M}\right.$ and $\left.c_{H}=1 \mathrm{M}\right)$. Play speed is $2 \times$. Movie S3: Top and side views of the numerical simulation results for the concentration of colloidal particles in the presence of the $\mathrm{NaCl}$ concentration gradient $\left(c_{L}=10 \mu \mathrm{M}\right.$ and $c_{H}=1$ M) with $\Gamma_{p}=450 \mu \mathrm{m}^{2} / \mathrm{s}$ and $\Gamma_{w}=780 \mu \mathrm{m}^{2} / \mathrm{s}$. Play speed is $30 \times$.

Movie S4: Top and side views of the numerical simulation results for the extraction of preconcentrated colloidal particles in the presence of the concentration gradient of K-acetate $\left(c_{L}=10 \mu \mathrm{M}\right.$ and $c_{H}=1 \mathrm{M}$ ) with $\Gamma_{p}=-40 \mu \mathrm{m}^{2} / \mathrm{s}$ and $\Gamma_{w}=-50 \mu \mathrm{m}^{2} / \mathrm{s}$. Play speed is $180 \times$.

Movie S5: Top and side views of the numerical simulation results for extraction of preconcentrated colloidal particles in the presence of the K-acetate concentration gradient $\left(c_{L}=\right.$ $10 \mu \mathrm{M}$ and $c_{H}=1 \mathrm{M}$ ) containing $0.02 \%$ Pluronic F-127 with $\Gamma_{p}=-70 \mu \mathrm{m}^{2} / \mathrm{s}$ and $\Gamma_{w}=-48$ $\mu \mathrm{m}^{2} / \mathrm{s}$. Play speed is $180 \mathrm{x}$.

Movie S6: Concentration and extraction of colloidal particles (1- $\mu \mathrm{m}$ carboxylated polystyrene) in three consecutive cycles depending on the configuration of the solute concentration gradient. In each cycle, particles are concentrated in the presence of the $\mathrm{NaCl}$ concentration gradient $\left(c_{L}\right.$ 
$=10 \mu \mathrm{M}$ and $\left.c_{H}=1 \mathrm{M}\right)$ for $1 \mathrm{~h}$ and extracted in the presence of the $\mathrm{K}$-acetate concentration gradient $\left(c_{L}=10 \mu \mathrm{M}\right.$ and $\left.c_{H}=1 \mathrm{M}\right)$ with $0.02 \%$ Pluronic F-127 for 15 min. Play speed is $800 \times$. Movie S7: Migration of preconcentrated particle mixture composed of red-fluorescent 200-nm and blue-fluorescent 620 -nm polystyrene induced by the Brownian motion in the absence of concentration gradient of solutes $\left(\mathrm{NaCl}, c_{L}=c_{H}=10 \mathrm{mM}\right)$. Play speed is $60 \times$.

Movie S8: Migration of preconcentrated particle mixture composed of red-fluorescent 200-nm and blue-fluorescent 620 -nm polystyrene in the presence of concentration gradients of (a) Kacetate $\left(c_{L}=0 \mathrm{M}\right.$ and $\left.c_{H}=1 \mathrm{M}\right)$, (b) Na-acetate $\left(c_{L}=0 \mathrm{M}\right.$ and $\left.c_{H}=1 \mathrm{M}\right)$, and (c) a mixture of $\mathrm{K}$-acetate $\left(c_{L}=0 \mathrm{M}\right.$ and $\left.c_{H}=200 \mathrm{mM}\right)$ and Na-acetate $\left(c_{L}=0 \mathrm{M}\right.$ and $\left.c_{H}=800 \mathrm{mM}\right)$. The result shows another experimental set for the separation of different particle mixture, as shown in Figure 3. Play speed is $60 \times$.

Movie S9: Migration of preconcentrated single particle type with red-fluorescent 200-nm and blue fluorescent 620 -nm polystyrene in the presence of concentration gradients of (a) K-acetate $\left(c_{L}=0 \mathrm{M}\right.$ and $\left.c_{H}=1 \mathrm{M}\right)$ and (b) Na-acetate $\left(c_{L}=0 \mathrm{M}\right.$ and $\left.c_{H}=1 \mathrm{M}\right)$. Play speed is $60 \times$. 


\section{References}

1. Shin, S.; Um, E.; Sabass, B.; Ault, J. T.; Rahimi, M.; Warren, P. B.; Stone, H. A., SizeDependent Control of Colloid Transport via Solute Gradients in Dead-End Channels. Proc. Natl. Acad. Sci. U.S.A. 2016, 113, 257-261.

2. Kar, A.; Chiang, T.-Y.; Ortiz Rivera, I.; Sen, A.; Velegol, D., Enhanced Transport into and out of Dead-End Pores. ACS Nano 2015, 9, 746-753.

3. Shin, S.; Ault, J. T.; Feng, J.; Warren, P. B.; Stone, H. A., Low-Cost Zeta Potentiometry Using Solute Gradients. Adv. Mater. 2017, 29, 1701516-n/a.

4. Gregory, J., Particles in Water: Properties and Processes. IWA Pub Taylor \& Francis Group: London 2005, 63-92. 